\title{
Evaluation of the role of an antioxidant gene in NSC-34 motor neuron-like cells as a model of a motor neuron disease
}

\author{
A.R. Alrafiah ${ }^{1,2}$ \\ ${ }^{1}$ Department of Medical Laboratory Technology, Faculty of Applied Medical Sciences, Jeddah, Saudi Arabia \\ ${ }^{2}$ Neuroscience Research Unit, King Abdulaziz University, Faculty of Medicine, Branch of Sulaymaniyah, Jeddah, Saudi Arabia
}

[Received: 18 June 2018; Accepted: 10 July 2018]

Background: Spinal muscular atrophy is a rare genetic disease, which primarily affects motor neurons and predominantly occurs in children. To date, alternatives for the treatment of the disease have been controversial. Spinal muscular atrophy has a multi-factorial aetiology, with mitochondrial oxidative stress considered as the crucial pathogenic mechanism. To determine the mechanisms underlying the loss of motor neurons, NSC-34 motor neuron-like cells are often used as an in vitro model of spinal muscular atrophy. As plastin 3 (PLS3) has been demonstrated as a modifier of spinal muscular atrophy, the aim of the current study was to evaluate the neuroprotective effect of PLS3 in NSC-34 cells.

Materials and methods: Plastin 3 was overexpressed in human embryonic kidney 293 T cells and NSC-34 cells via lentiviral transduction. NSC-34 cells transduced with a lentiviral vector carrying the gene for LacZ $\beta$-galactosidase served as a control. Oxidative stress was then induced by depriving cells of serum, and the protective effect of PLS3 was assessed using a cellular reactive oxygen species detection assay. Results: While PLS3 was successfully overexpressed in human embryonic kidney $293 T$ cells and NSC-34 cells, upregulation of this protein did not significantly decrease oxidative stress in serum-deprived NSC-34 cells relative to controls.

Conclusions: Plastin 3 overexpression in NSC-34 cells did not elicit an antioxidative effect following serum deprivation. (Folia Morphol 2019; 78, 1: 1-9)

Key words: oxidative stress, plastin 3, NSC-34 cells, lentiviral vectors, spinal muscular atrophy

\section{INTRODUCTION}

Spinal muscular atrophy (SMA) - a destructive motor neuron (MN) disorder which occurs in infancy - is primarily caused by survival motor neuron $1 \mathrm{gene}$ (SMN1) mutations. SMN1 and SMN2 are nearly identical genes encoding the survival motor neuron (SMN) protein $[16,23]$. Mutations in the SMN1 gene lead to the reduction or loss of SMN protein expression, causing motor axon defects and MN death [3, 16].
Generally, reduced SMN protein expression is believed to cause the death of lower motor neurons, thereby inducing denervation and skeletal muscle atrophy [3]. The major clinical characteristic of SMA is the selective and progressive loss of MNs via apoptosis, although the factors, which trigger this loss, remain unknown [9]. Multiple interacting factors have been implicated in MN lesions, including mitochondrial dysfunction, excitotoxicity, protein aggregation and

Address for correspondence: A.R. Al-Rafiah, PhD, MSc, BSc, Assistant Professor, Medical Laboratory Technology Department, Faculty of Applied Medical Sciences, Head of Neuroscience Research Unit, Medical School, King Abdulaziz University, P.O. Box 80200, Jeddah 21589, KSA, tel: +966126400000/ext: 23495, e-mail: aalrafiah@kau.edu.sa 
misfolding, defective axonal transport, dysregulated transcription and RNA processing, apoptosis, and oxidative stress $[5,16,17]$.

While SMA has traditionally been classified as a pure lower MN disease, it is becoming clear that supplementary cells and tissue types are particularly susceptible to decreased SMN expression. Current therapeutic strategies focus on increasing SMN expression; however, developing an effective treatment for all affected cells remains a challenge. Targeting cells other than MNs in order to successfully provide therapy for SMA is possible via the intramuscular administration of adeno-associated virus serotype 9 (AAV9) carrying the $S M N$ gene, which can enter the spinal cord via retrograde transport and transduce multiple organs in peripheral tissues. Indeed, gene therapy approaches using AAV9-SMN vectors have entered phase I clinical trials and are highly promising. However, limitations associated with this treatment highlight the need for combined therapies that rescue both MN function and increase SMN levels. As such, the identification and characterisation of SMA-related pathologies that occur across tissues and affected cells is crucial for the development of novel SMA therapeutic strategies $[2,16]$.

Oxidative stress is an instrumental factor in the pathogenesis of many neurodegenerative diseases [14], and typically occurs when a cell fails to cope with excess levels of reactive oxygen species (ROS), resulting in toxic effects facilitated by peroxides and free radicals. ROS is formed via uncoupled nitric oxide synthase and nicotinamide adenine dinucleotide phosphate oxidase [26]. Antioxidants are important for the removal of ROS before they become problematic. If ROS is not removed, chronic oxidative stress can lead to DNA damage and the subsequent promotion of cell death [26].

The involvement of oxidative stress in SMA pathogenesis remains unclear. For instance, nicotinamide adenine dinucleotide phosphate levels are reportedly elevated in the cardiac tissue of (SMN $\Delta 7$ ) mice, but are unchanged in the spinal cord [27]. Moreover, severe mitochondrial oxidative stress is elicited by SMN breakdown in human embryonic stem cell-derived MN progenitors, and antioxidant treatment with $\mathrm{N}$-acetylcysteine prevents the apoptotic death of MNs [2].

Oprea et al. [23] reported plastin 3 (PLS3), as an SMA modifier, qualifying it as a promising therapeutic target. In agreement with this, PLS3 gene overexpression has been shown to refine axonal outgrowth in SMN-deficient MNs in a zebrafish model of SMA as well as in MNs cultured from mouse embryos $[16,17]$. Several studies have reported an actin-independent role of PLS3 in SMA modification [1, 17]; however, investigations on the potential protective role of PLS3 against oxidative stress have not been performed. Some reports have described the involvement of oxidative damage in the neurodegenerative process of SMA, and neuroprotection is considered to be an effective treatment option for many central nervous system disorders, including neurodegenerative diseases such as SMA $[5,17]$. Therefore, a therapeutic strategy involving antioxidant gene delivery could provide significant protection for MNs in SMA [16]. This study is, to the best of our knowledge, the first to address whether overexpression of PLS3 using a lentiviral vector (LV) in an in vitro model of SMA could reduce oxidative stress and thus protect MN-like cells.

\section{MATERIALS AND METHODS}

\section{Cell culture}

Ahybrid mouse MN-neuroblastoma cell line, NSC-34, was purchased from Cedarlane Laboratories (CLU 140-A; thru Tebu-Bio, Le Perray en Yvelines, France) and cultured as previously reported [19]. The human embryonic kidney cell line, HEK293T, (293T ATCC CRL-3216) was also used. All cell lines were cultured in tissue culture flasks (CELLSTAR, Greiner, Germany) in Dulbecco's Modified Eagle's Medium (Lonza, BE12-741F) with $10 \%$ heat-inactivated foetal bovine serum (Biosera, FB-1001), $100 \mathrm{U} / \mathrm{mL}$ of penicillin and $100 \mu \mathrm{g} / \mathrm{mL}$ streptomycin (Invitrogen, Paisley, UK). Cells were incubated at $37^{\circ} \mathrm{C}$ and in $5 \% \mathrm{CO}_{2}$ with a $95 \%$ humidified air atmosphere. Cells were subcultivated at a ratio of 1:8 twice weekly by removing medium, adding $2 \mathrm{~mL}$ of trypsin (Sigma, 9002-07-7) supplemented with $0.5 \mathrm{mM}$ ethylenediaminetetraacetic acid (EDTA, Sigma, 6381-92-6) in phosphate-buffered saline (PBS), and incubating for several minutes. When cells detached, the enzymatic activity of trypsin was halted by adding excess culturing medium. All experiments were performed on passage 15-20. Once cells reached approximately $80 \%$ confluency, they were either harvested or left for passaging.

\section{Lentiviral vectors}

All LVs were provided by Prof. Mimoun Azzouz at the University of Sheffield, United Kingdom and were generated in his laboratory. LVs expressing PLS3 were constructed by extracting PLS3 gene out of the vector 
(-pc'DNA3.1-PLS3-V5-His6) and then cloned it into the (SIN-PGK-CPPT-GDNF-WHV) LV; LV titre was $2.55 \times 10^{8}$ $\mathrm{TU} / \mathrm{mL}$. The LV expressing LacZ was generated using cDNA for "beta-galactosidase (LacZ)" and "humanistic GDNF encompassing Kozak sequence (636 bp fragment, position 1-4851 and 1-151)" (GenBank L19062 and L19063 accession numbers), thus preventing secretion of the trophic factor [11]. These cDNAs were replicated in the SIN-W-PGK transfer vector [12].

\section{Transduction of HEK293T and NSC-34 cells with LV-PLS3}

HEK239T and NSC-34 cells were plated in a 6-well plate at a density of 150,000-250,000 cells/well in $2 \mathrm{~mL}$ of medium and incubated overnight at $37^{\circ} \mathrm{C}$ in a $\mathrm{CO}_{2}$ incubator. The $\mathrm{LV}$ volume required for transduction was calculated and cells were transduced at multiplicity of infection (MOI) of 5, 20, and 40 . The calculated LV volume was added to each well after the removal of $1 \mathrm{~mL}$ of medium. After $6 \mathrm{~h}$ of incubation, wells were supplemented with $1 \mathrm{~mL}$ of medium and incubated for an additional 3-5 days.

Five days post-transduction, cells were harvested for protein analysis. Non-transduced (NT) cells served as a negative control, and an antibody specific for human PLS3 protein was used. Protein extraction was accomplished by the addition of cell lysis solution (RIPA buffer) containing $50 \mathrm{mM}$ Tris- $\mathrm{HCl} \mathrm{pH} \mathrm{8,}$ $150 \mathrm{mM} \mathrm{NaCl}, 2 \mathrm{mM}$ EDTA, 1\% Igepal CA630 (Sigma), and $0.1 \%$ sodium dodecyl sulphate (SDS) (Sigma) supplemented with $1 \%$ protease inhibitor cocktail (Sigma). The cell pellet was resuspended in 50-150 $\mu \mathrm{L}$ of the lysis solution and incubated for $30 \mathrm{~min}$ on ice. Cell debris was separated from protein content by centrifugation at $13,000 \times \mathrm{g}$ for $10 \mathrm{~min}$, after which the supernatant was transferred into a clean $1.5 \mathrm{~mL}$ tube. Extracted protein was measured using a Bicinchoninic Acid Protein Assay kit (Thermo Fisher, Waltham, MA, USA).

\section{Western blotting}

Denatured protein samples were run $(20 \mu \mathrm{g}$ protein/sample) on $12 \%$ SDS-polyacrylamide gels (BioRad; 4561043EDU) and electro-transferred onto a polyvinylidene fluoride membrane (Thermo Fisher).

The following primary antibodies were utilised to probe the membrane: rabbit anti-mouse (polyclonal antibody-middle region) PLS3 (PA5-27883) (Thermo Fisher, 1/500) and anti-mouse alpha-tubulin (Calbiochem, 1/1000). Secondary antibodies included goat anti-rabbit IgG H\&L (HRP) (Abcam ab45769, $1 / 1000$ ). The membrane was developed using the enhanced chemiluminescence plus detection kit (Biological industries, 20-500-120), and signals were visualised using G-Box (Sygene) and Chemilmager software (Alpha Innotech). Alpha-tubulin was used as a loading control. The density of each band was integrated and the ratio of the densitometric values of each sample was obtained. Western blotting was performed in triplicate. Variation in protein expression was evaluated using Chemilmager software to compare samples and controls.

\section{Immunocytochemistry}

Immunofluorescence staining was performed on HEK293T and NSC-34 cells. Using a 6-well plate, at a density of 50,000 cells per well the cells were plated onto glass coverslips. Both cell lines were transduced with LV-PLS3. Five days after transduction, cells were washed with PBS and fixed in $4 \%$ paraformaldehyde in PBS ( $\mathrm{pH} \mathrm{7.3)} \mathrm{for} 15 \mathrm{~min}$ at $25^{\circ} \mathrm{C}$. Thereafter, cells were washed with Dulbecco's PBS and stored at $4^{\circ} \mathrm{C}$ until the staining procedure was performed. Cell membranes were permeabilised by incubating the cells in blocking solution $(0.3 \%$ Triton $\mathrm{X}-100$ and $10 \%$ goat serum in PBS) for $2 \mathrm{~h}$ at $25^{\circ} \mathrm{C}$. The blocking solution was removed and cells were incubated in anti-rabbit PLS3 (1:500; ab45769) overnight. The next day, following three 10-min washes in PBS, cells were incubated in anti-rabbit lgG Alexa Fluor 568 secondary antibody for $1 \mathrm{~h}$ in the dark at $25^{\circ} \mathrm{C}$. Finally, cells were washed 10 min in PBS and mounted using Vectashield hard mounting medium on glass slides with 4',6-diamidino-2-phenylindole (DAPI; D9542 Sigma). Confocal images of selected fields were collected using a Leica SP5 microscope system at $63 \times$ magnification and were processed using Image J software. Approximately 20 images were taken per slide; representative images are presented in Figures 1 and 2.

\section{Cell viability assay and oxidative stress assessment}

NSC-34 cells were plated in 96-well plates at a density of 20,000 cells/well. Plates contained NT cells and LV-LacZ-transduced (20 MOI) or LV-PLS3-transduced $(20 \mathrm{MOI})$ cells. Each condition comprised 9 replicates.

In order to induce oxidative stress, NSC-34 cells were deprived of serum for $6 \mathrm{~h}$. To assess oxidative stress, dichlorodihydrofluorescein (DCF) dye (10 mM) (Thermo Fisher) was added to the NSC-34 cells at 


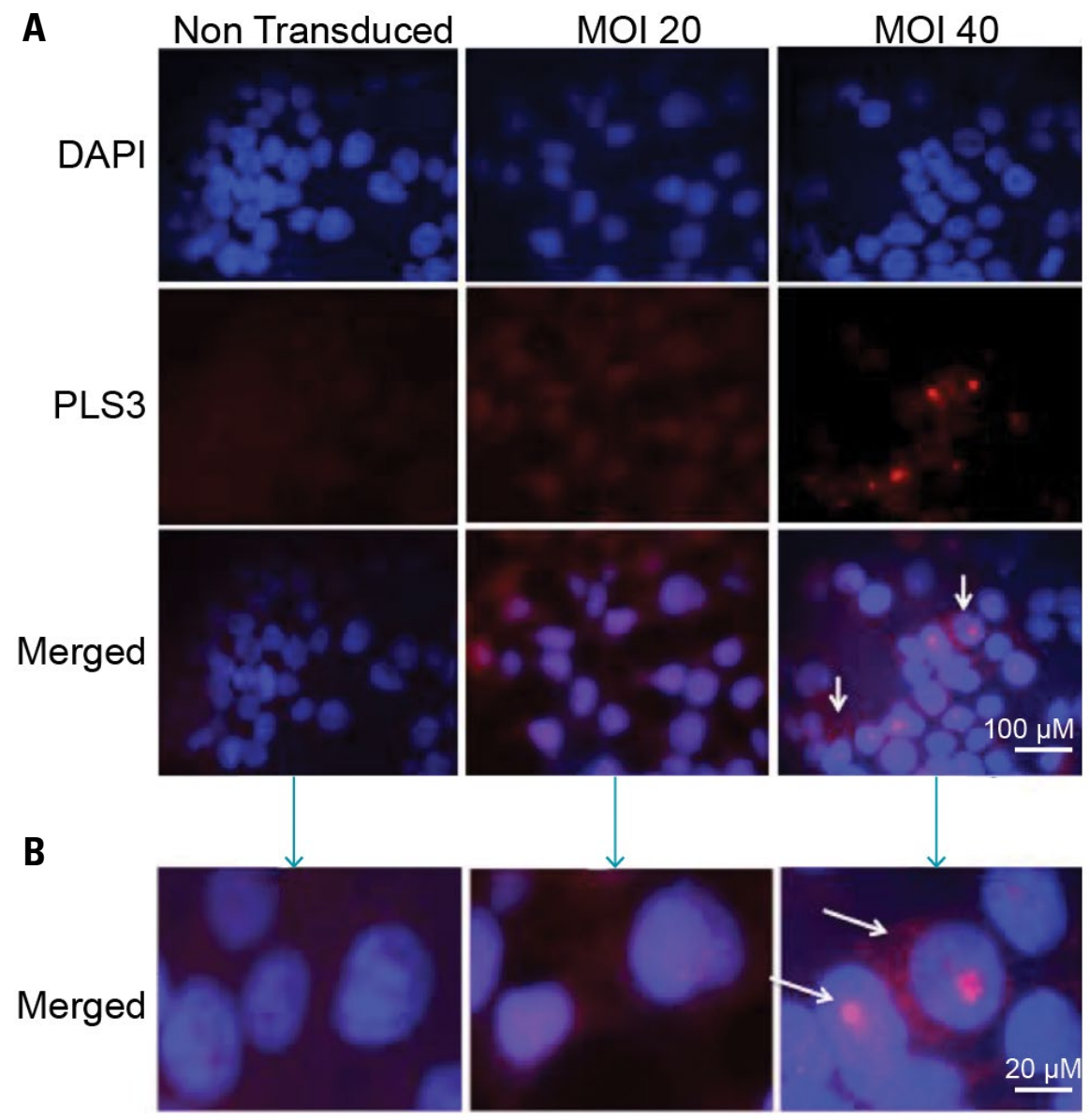

Figure 1. A, B. Immunofluorescent validation of lentiviral vector (LV)-plastin 3 (PLS3) transduction in HEK293T cells. LV-PLS3 was introduced to HEK293T cells at multiplicity of infection (MOI) levels of either 20 or 40. Non-transduced cells were immunostained and served as a negative control. Nuclei are counterstained with DAPI (blue) and PLS3 is indicated in red. The white arrows indicate PLS3 expression in both the nucleoli and the cytoplasm. Scale bar; $100 \mu \mathrm{m}(\mathrm{A})$ and $20 \mu \mathrm{m}$ (B).

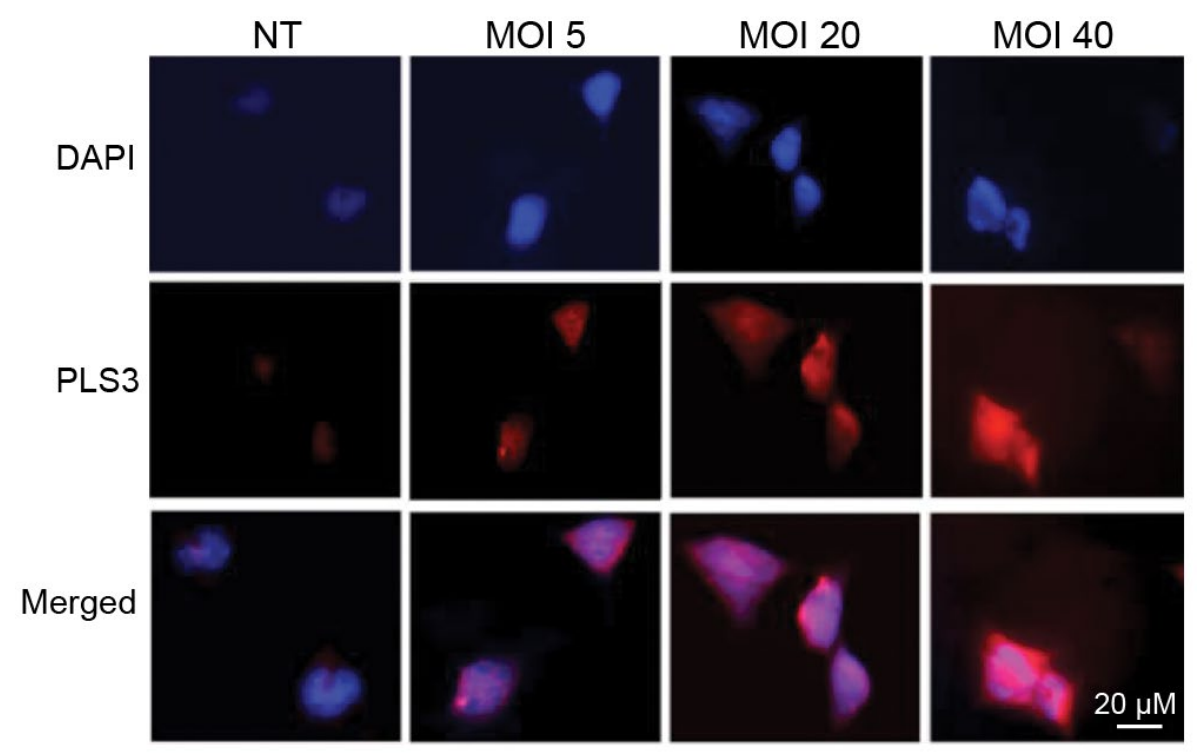

Figure 2. Lentiviral vector (LV)-mediated plastin 3 (PLS3) overexpression in NSC-34 cells assessed by immunofluorescence. NSC-34 cells were transduced with LV-PLS3 at various multiplicities of infection (MOIs, 5, 20, and 40). Non-transduced (NT) cells were included as negative controls. Nuclei are counterstained with DAPI (blue) and PLS3 is indicated in red. The scale bar $(20 \mu \mathrm{m})$. 
a final concentration of $5 \mu \mathrm{M}$. An initial reading was acquired prior to the dye addition to account for background fluorescence. Oxidative stress was calculated by monitoring DCF fluorescence acceleration (emission at $530 \mathrm{~nm}$ and excitation at $485 \mathrm{~nm}$ ). Toxicity of the assay was checked through the addition of ethidium homodimer-1 (EthD-1) dye (Invitrogen), together with the DCF dye, at an absolute $0.33 \mu \mathrm{M}$ concentration. Fluorescence was measured at an excitation of $530 \mathrm{~nm} / \mathrm{emission} 645 \mathrm{~nm}$ using a Sian programme on the fusion microplate reader (Perkin Elmer). LVs were added $1 \mathrm{~h}$ after serum withdrawal, at the beginning of the experiment.

In order to analyse these experiments, the average blank fluorescence value was subtracted from each well for both DCF and EthD-1 data. Data was normalised by dividing DCF fluorescence by EthD-1 fluorescence. Ten plates were assayed and statistical analysis was performed after normalising the results of each plate to the values obtained in NT cells.

Quantification of living cell numbers in the 96-well plates was performed using dilutions of 0.1 volumes reduction of $5 \mathrm{mg} / \mathrm{mL}$ MTT (Thermo Fisher) via cellular dehydrogenases. One dimethylformamide volume was added (DMF, SDS, $20 \%$ SDS in $50 \%$ DMF, pH 4.7) per well at $25^{\circ} \mathrm{C}$ and, after $1 \mathrm{~h}$ of incubation at $37^{\circ} \mathrm{C}$, wells were placed on a shaking incubator for $1 \mathrm{~h}$. Absorbance was assessed at $595 \mathrm{~nm}$.

\section{Statistical analysis}

Data were assessed using a one-way ANOVA and results are presented as the average measurements per plate \pm standard deviation (SD).

Graph-Pad Prism software version 7.0 was used to analyse the data. A one-way ANOVA was utilised to compare the variance between untreated and treated cells. $\mathrm{P}<0.05$ was considered significantly different.

\section{RESULTS}

Lentiviral vectors mediate efficient overexpression of PLS3 in cell lines

Human embryonic kidney (HEK293T) and NSC-34 MN-like cells transduced with an LV carrying the PLS3 gene (LV/PLS3) exhibited a significant rise in PLS3 expression compared to NT control cells.

\section{PLS3 protein levels in HEK293T cells transduced with LV-PLS3}

Transduction of HEK293T cells was performed using the LV-PLS3 vector at different multiplicities
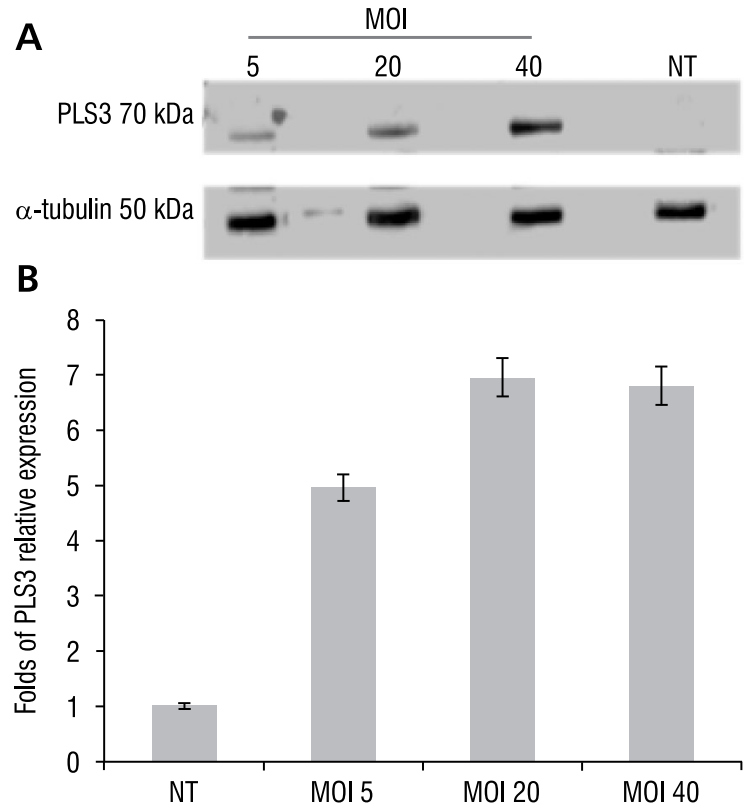

Figure 3. Western blot validation of lentiviral vector (LV)-plastin 3 (PLS3) transduction efficiency in HEK293T cells. A. Western blot of PLS3 protein expression in HEK293T cells transduced with LV-PLS3 $(5,20$, and 40$)$ multiplicities of infection (MOls). Non-transduced (NT) cells served as a negative control. WB membrane was probed with antibody against human PLS3. Alpha-tubulin served as loading control; B. Densitometry analysis of PLS3 levels in transduced cells with different LV-PLS3 MOls $(5,20$, and 40$)$ normalised to NT cells.

of infection (MOIs; 5,20 , and 40). The experiment was repeated three times $(n=3)$ and demonstrated that LV-PLS3 transduction successfully induced PLS3 overexpression in HEK293T cells (Fig. 3). Western blot analysis (Fig $3 \mathrm{~A}$ ) revealed the appearance of a protein band (70-kDa), indicative of PLS3 in all three conditions. A 50-kDa band was detected using western immunoblotting for $\alpha$-tubulin, which served as a loading control. In NT cells, endogenous PLS3 expression was undetectable. Densitometry analysis of western blots revealed a correlation between increased transgene PLS3 expression and the $\mathrm{MOI}$ of the LV used for transduction. PLS3 expression was maximum 5 days after transduction with $\mathrm{MOI} 20$, showing a 7-fold increase compared to NT cells (Fig. 3B). Moreover, a previous study reported no signs of toxicity or any apparent differences in cellular growth rate, even after transduction with higher MOIs (data not provided) [13].

\section{Transduction efficiency of LV-PLS3 in HEK293T cells}

Immunofluorescence was performed to evaluate the expression and localisation of PLS3 in HEK293T cells $72 \mathrm{~h}$ after transduction with (LV-PLS3; MOls 
5 and 20) compared to NT HEK293T cells (Fig. 1). The experiment was performed in triplicate $(n=3)$. The results summarised in Figure 1 indicate that approximately $80 \%$ of the LV-PLS3 transduced cells with MOI 20 overexpressed human PLS3. PLS3 localisation was observed in a granular staining pattern, covering an area within the cytoplasm and nucleus. This experiment confirmed that the LV-PLS3 vector was capable of mediating PLS3 gene delivery and expression in vitro.

\section{LV-PLS3 transduction efficiency in NSC-34 cells}

After verifying the functionality of LV-PLS3 in HEK293T cells, the same LV was used to transduce NSC-34 MN-like cells in order to investigate PLS3 effect in an in vitro SMA model. Immunofluorescence was first used to assess the expression of PLS3 5 days after transduction at different LV-PLS3 MOI levels (5, 20 , and 40) compared to non-transduced NSC-34 cells. The experiment was repeated 3 times $(n=3)$. PLS3 localisation was observed throughout NSC-34 cells and was associated with the cytoplasm and nucleus (Fig. 1). Interestingly, this staining pattern differed from that observed in HEK293T cells, which was granular in nature (Figs. 1, 2). This observation may be due to differences in cell type. This experiment confirmed functionality and efficacy of the LV-PLS3 vector in expressing PLS3 in NSC-34 MN-like cells.

\section{Oxidative stress measurement in PLS3-overexpressing NSC-34 cells}

In order to determine whether PLS3 overexpression reduced oxidative stress and thus protected cells against subsequent damage, NT cells and NSC-34 cells transduced to overexpress PLS3 or LacZ were cultured under serum-deprived conditions and subjected to the DCF assay. A separate set of NT control cells cultured in serum (basal condition) were subjected to the same DCF assay. DCF is a lipophilic cell-permeant dye which, upon entering a cell, loses its acetate groups via intracellular esterases. This action renders the dye hydrophilic and thus unable to leave the cell, making it suitable for measuring intracellular oxidative stress. Using this approach, serum-deprived NT cells exhibited significant increases in intracellular levels of oxidised DCF when compared to NT cells under basal conditions ( $p<0.05$; Fig. 4). This experiment also revealed that PLS3-overexpression in serum-deprived NSC-34 cells did not significantly reduce oxidative stress relative to LV-LacZ-transduced and NT cells

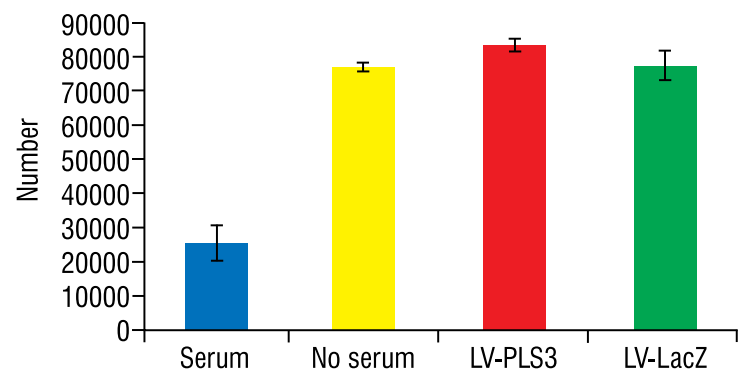

Figure 4. Oxidative stress levels in NSC-34 cells as assessed by a dichlorodihydrofluorescein assay after serum deprivation. Overexpression of plastin 3 (PLS3) in NSC-34 cells (red bar) did not significantly decrease serum deprivation-induced oxidative stress when compared to lentiviral vector (LV-LacZ)-transduced (green bar) and non-transduced (NT) cells (yellow bar). No significant difference in oxidative stress was observed in NT cells under basal conditions (blue bar, data not provided).

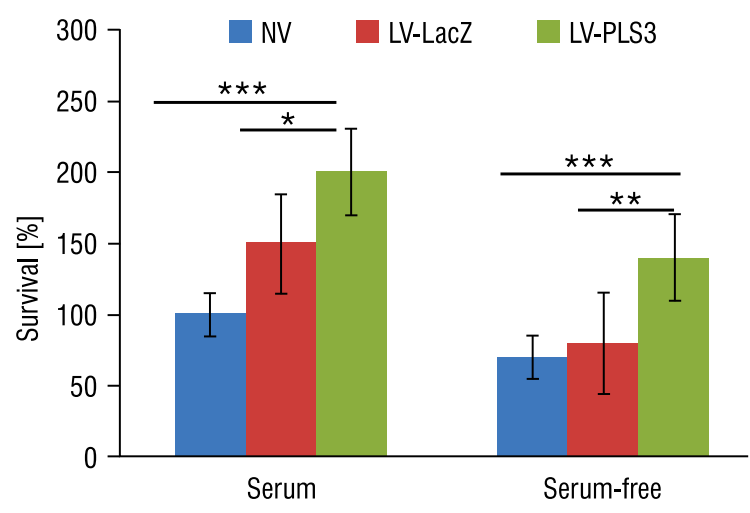

Figure 5. NSC-34 cell survival as determined by a cell metabolic activity assay. The 3'-(4',5'-dimethylthiazol-2-yl)-2',5'-diphenyltetrazolium bromide (MTT) assay was used to determined NSC-34 cell survival 5 days post-transduction with lentiviral vector (LV)-plastin 3 (PLS3) or LV-LacZ (multiplicity of infection $=20$ ) under basal conditions and after oxidative stress induction (two-way ANOVA, Bonferroni's posthoc test, $\mathrm{p}<0.05, \mathrm{n}=5$, mean \pm SEM); ${ }^{*} \mathrm{p}<0.05 ;{ }^{* *} \mathrm{p}<0.01$; *** $p<0.001$.

grown under the same conditions. This demonstrates that PLS3 overexpression was not sufficient to protect NSC-34 MN-like cells against oxidative damage.

\section{Cell metabolic activity assay}

After the DCF assay, a cell metabolic activity assay (3'-(4',5'-dimethylthiazol-2-yl)-2', 5'-diphenyltetrazolium bromide [MTT]) was performed on the same cultures in order to justify plating variability; data obtained by the MTT assay were normalised in accordance with the number of cells.

After oxidative stress induction, the durability of cells transduced with LV-PLS3 was observed to increase by approximately $92 \%(p<0.001, n=4)$ in comparison to NT cells and $61 \%(p<0.01, n=4)$ in comparison to LV-LacZ-transduced cells (Fig. 5). 
Previous studies have confirmed that $6 \mathrm{~h}$ of menadione administration in a redox cycling system is sufficient to increase ROS within numerous subcellular chambers [20]. In this study, PLS3 overexpression in serum-deprived NSC-34 cells promoted cell survivability following menadione administration; however, the increase in viability was not significantly different when compared to serum-deprived NT cells. One possible explanation for the lack of significance is the elevated toxicity and cell loss (76\%) observed in NT cells. As NSC-34 cells are specifically susceptible to oxidative stress, cell death likely occurred prior to the redeeming effect of PLS3 upregulation. Furthermore, delivering $P L S 3$ under basal conditions resulted in a $68 \%$ increase in NSC-34 cell number $(p<0.001$, $\mathrm{n}=4$ ) compared to that of NT cells. Likewise, LV-PLS3 transduction under basal conditions increased NSC-34 cell counts by $35 \%(p<0.05, n=4)$ in comparison to cells transduced with LV-LacZ, and further resulted in a greater growth ratio.

\section{DISCUSSION}

This study attempted to assess the potential PLS3 anti-oxidative effect of in an in vitro SMA model. In order to do so, a lentivirus was used to confer stable PLS3 overexpression in NSC-34 cells. Oxidative stress was then induced by depriving cells of serum and the levels of intracellular oxidative mediators were assessed using a fluorimetric assay (DCF). LVs were selected as gene delivery vectors due to their ability to efficiently transfer genes to cells of the CNS in vitro and in vivo, and because they can transduce both dividing and non-dividing cells, including neurons [6]. In addition, it has been reported that LVs can efficiently deliver genes to different primary neuron types across a broad range of species, including humans, resulting in long-term gene expression $[8,15]$. Two cell lines were used (HEK293T and NSC-34) to assess LV transduction efficiency, and NSC-34 MN-like cells were further selected as an in vitro model of SMA. Ideally, SMA pathophysiology should be studied in in vitro cultures of primary MNs $[10,15,18,23]$. However, due to decreased MN culture yields, the NSC-34 cell line is considered a suitable alternative to $\mathrm{MN}$ cultures and has been widely used to model MN diseases [13]. Indeed, NSC-34 MN-like cells differentiate via a process resembling that of primary MNs, comprise extended neurites, and express $\mathrm{MN}$-specific markers [4].

The therapeutic potential of the PLS3 gene has previously been demonstrated using a tissue cul- ture-based example of SMN1-associated SMA [7, 23]. While the findings of the current study are in agreement with previous reports, this investigation revealed that viral delivery of PLS3 did not protect NSC-34 cells against serum deprivation-induced oxidative stress. The distribution of PLS3 in astrocytes and other neural cells should be considered when investigating the therapeutic potential of virally delivered PLS3 to MNs. Our previous research on the differential expression patterns of PLS3 provided histological evidence that PLS3 immunoreactivity is present in the mouse cerebrum and spinal cord [4]. This supports the hypothesis that direct gene transmission toward MNs may promote the development of protective strategies to counter oxidative stress. However, viral delivery of SMN into SMA pluripotent stem cell cultures (SMN-deficient) restored catalase levels to normal and reversed astrocyte activation, without changing the formation of the markers of the oxidative stress or the mitochondrial respiration. These results reveal that SMN insufficiency promotes the reactivity of astrocyte, but that this effect is not elicited via an oxidative stress-mediated process [25].

While several factors, including oxidative stress, contribute to various age-affiliated neurodegenerative disorders, their involvement in the pathogenesis of SMA remains unclear. One potential interpretation for this variation is the age of onset for various agelinked diseases, where oxidative stress could increase over time in order to alter neurons in a specific region. As SMA type I occurs in infancy, neurons are only exposed to stressful surroundings for a brief amount of time. Moreover, for the reduction of stress and cell loss, SMN-deficient cells may utilize endogenous mechanisms.

In the current study, PLS3 overexpression did not significantly reduce oxidative stress, nor did it obviously alter NSC-34 survival or morphology. These findings also reveal that other antioxidant genes associated with this pathway were unable to independently induce neuroprotection. One explanation for the lack of effect is the possibility that cells were not sufficiently mature, prohibiting the ability to reduce oxidative stress under the current circumstances. Furthermore, the use of primary MN cultures, rather than the NSC-34 cell line, would be more appropriate to elucidate mechanisms underlying cell protection within SMA and other MN disorders. Nonetheless, NSC-34 cells continue to be fitting for studies on neuroprotection [22] and neurotoxins [21], and to explore 
the outcomes of cerebrolysin [22]. As mentioned, NSC-34 cells display similar properties to MNs when exposed to conditions which promote maturation and differentiation [24]. Moreover, the NSC-34 cell line could be adopted to study the pathophysiological features of amyotrophic lateral sclerosis - another neurodegenerative $\mathrm{MN}$ disease. Thus, while this cell line was not ideal for the current study, it appears well-suited as a therapeutic screening tool and for specialised experiments.

\section{CONCLUSIONS}

In this study, PLS3 overexpression in NSC-34 MN-like cells did not readily reduce serum deprivation-induced oxidative stress levels. These findings do not, however, exclude the possibility that PLS3 exerts an anti-oxidative effect that could not be detected using the current experimental setup. Future investigations using other experimental designs that include primary mouse MNs or human induced pluripotent stem cell-derived MNs from patients with SMA will be useful to determine the full neuroprotective efficacy of PLS3.

\section{Acknowledgements}

I wish to thank Prof. Mimoun Azzouz in the Department of Neuroscience Sheffield Institute for Translational Neuroscience at the University of Sheffield, United Kingdom for kindly providing the lentiviral viruses.

\section{Funding}

This Project was funded by the Deanship of Scientific Research (DSR), at King Abdulaziz University, Jeddah, under grant no. G-200-290-39. The authors, therefore, acknowledge with thanks DSR for technical and financial support.

\section{REFERENCES}

1. Ackermann B, Kröber $S$, Torres-Benito $L$, et al. Plastin 3 ameliorates spinal muscular atrophy via delayed axon pruning and improves neuromuscular junction functionality. Hum Mol Genet. 2013; 22(7): 1328-1347, doi: 10.1093/ hmg/dds540, indexed in Pubmed: 23263861.

2. Acsadi G, Lee I, Li X, et al. Mitochondrial dysfunction in a neural cell model of spinal muscular atrophy. J Neurosci Res. 2009; 87(12): 2748-2756, doi: 10.1002/jnr.22106, indexed in Pubmed: 19437551.

3. Alías L, Bernal S, Fuentes-Prior P, et al. Mutation update of spinal muscular atrophy in Spain: molecular characterization of 745 unrelated patients and identification of four novel mutations in the SMN1 gene. Hum Genet. 2009; 125(1): 29-39, doi: 10.1007/s00439-008-0598-1, indexed in Pubmed: 19050931.
4. Alrafiah A, Karyka E, Coldicott I, et al. Plastin 3 promotes motor neuron axonal growth and extends survival in a mouse model of spinal muscular atrophy. Mol Ther Methods Clin Dev. 2018; 9: 81-89, doi: 10.1016/j. omtm.2018.01.007, indexed in Pubmed: 29552580.

5. Azzouz M, Le T, Ralph GS, et al. Lentivector-mediated SMN replacement in a mouse model of spinal muscular atrophy. J Clin Invest. 2004; 114(12): 1726-1731, doi: 10.1172/ JCI22922, indexed in Pubmed: 15599397.

6. Azzouz M, Martin-Rendon E, Barber RD, et al. Multicistronic lentiviral vector-mediated striatal gene transfer of aromatic L-amino acid decarboxylase, tyrosine hydroxylase, and GTP cyclohydrolase I induces sustained transgene expression, dopamine production, and functional improvement in a rat model of Parkinson's disease. J Neurosci. 2002; 22(23): 10302-10312, indexed in Pubmed: 12451130.

7. Bernal S, Also-Rallo E, Martínez-Hernández R, et al. Plastin 3 expression in discordant spinal muscular atrophy (SMA) siblings. Neuromuscul Disord. 2011; 21(6): 413-419, doi: 10.1016/j.nmd.2011.03.009, indexed in Pubmed: 21546251.

8. Bienemann AS, Martin-Rendon E, Cosgrave AS, et al. Long-term replacement of a mutated nonfunctional CNS gene: reversal of hypothalamic diabetes insipidus using an EIAV-based lentiviral vector expressing arginine vasopressin. Mol Ther. 2003; 7(5 Pt 1): 588-596, indexed in Pubmed: 12718901.

9. Burghes AHM, Beattie CE. Spinal muscular atrophy: why do low levels of survival motor neuron protein make motor neurons sick? Nat Rev Neurosci. 2009; 10(8): 597-609, doi: 10.1038/nrn2670, indexed in Pubmed: 19584893.

10. Cashman NR, Durham HD, Blusztajn JK, et al. Neuroblastoma $x$ spinal cord (NSC) hybrid cell lines resemble developing motor neurons. Dev Dyn. 1992; 194(3): 209-221, doi: 10.1002/aja.1001940306, indexed in Pubmed: 1467557.

11. Choi-Lundberg DL, Lin Q, Chang YN, et al. Dopaminergic neurons protected from degeneration by GDNF gene therapy. Science. 1997; 275(5301): 838-841, indexed in Pubmed: 9012352.

12. Déglon N, Tseng JL, Bensadoun JC, et al. Self-inactivating lentiviral vectors with enhanced transgene expression as potential gene transfer system in Parkinson's disease. Hum Gene Ther. 2000; 11(1): 179-190, doi: 10.1089/10430340050016256, indexed in Pubmed: 10646649.

13. Eaton SL, Roche SL, Llavero Hurtado M, et al. Total protein analysis as a reliable loading control for quantitative fluorescent Western blotting. PLoS One. 2013; 8(8): e72457, doi: 10.1371/journal.pone.0072457, indexed in Pubmed: 24023619.

14. Federico A, Cardaioli E, Da Pozzo P, et al. Mitochondria, oxidative stress and neurodegeneration. J Neurol Sci. 2012; 322(1-2): 254-262, doi: 10.1016/j.jns.2012.05.030, indexed in Pubmed: 22669122.

15. Hall CE, Yao Z, Choi M, et al. Progressive motor neuron pathology and the role of astrocytes in a human stem cell model of VCP-related ALS. Cell Rep. 2017; 19(9): 1739-1749, doi: 10.1016/j.celrep.2017.05.024, indexed in Pubmed: 28564594. 
16. Hamilton G, Gillingwater TH. Spinal muscular atrophy: going beyond the motor neuron. Trends Mol Med. 2013; 19(1): 40-50, doi: 10.1016/j.molmed.2012.11.002, indexed in Pubmed: 23228902.

17. Hosseinibarkooie $S$, Peters $M$, Torres-Benito $L$, et al. The power of human protective modifiers: PLS3 and CORO1C unravel impaired endocytosis in spinal muscular atrophy and rescue SMA phenotype. Am J Hum Genet. 2016; 99(3): 647-665, doi: 10.1016/j.ajhg.2016.07.014, indexed in Pubmed: 27499521.

18. Kanjilal B, Keyser BM, Andres DK, et al. Differentiated NSC-34 cells as an in vitro cell model for VX. Toxicol Mech Methods. 2014; 24(7): 488-494, doi: 10.3109/15376516. 2014.943442, indexed in Pubmed: 25045830.

19. Madji Hounoum B, Blasco H, Nadal-Desbarats L, et al. Analytical methodology for metabolomics study of adherent mammalian cells using NMR, GC-MS and LC-HRMS. Anal Bioanal Chem. 2015; 407(29): 8861-8872, doi: 10.1007/ s00216-015-9047-x, indexed in Pubmed: 26446897.

20. Madji Hounoum B, Vourc'h P, Felix R, et al. NSC-34 motor neuron-like cells are unsuitable as experimental model for glutamate-mediated excitotoxicity. Front Cell Neurosci. 2016; 10: 118, doi: 10.3389/fncel.2016.00118, indexed in Pubmed: 27242431.

21. Maier O, Böhm J, Dahm M, et al. Differentiated NSC-34 motoneuron-like cells as experimental model for cholinergic neurodegeneration. Neurochem Int. 2013; 62(8): 1029-1038, doi: 10.1016/j.neuint.2013.03.008, indexed in Pubmed: 23562846.
22. Nanou A. Gene therapy approaches to evaluate neuroprotection from oxidation stress in experimental models of amyotrophic lateral sclerosis. University of Sheffield. 2012.

23. Oprea GE, Kröber S, McWhorter ML, et al. Plastin 3 is a protective modifier of autosomal recessive spinal muscular atrophy. Science. 2008; 320(5875): 524-527, doi: 10.1126/ science.1155085, indexed in Pubmed: 18440926.

24. Park GH, Maeno-Hikichi Y, Awano T, et al. Reduced survival of motor neuron (SMN) protein in motor neuronal progenitors functions cell autonomously to cause spinal muscular atrophy in model mice expressing the human centromeric (SMN2) gene. J Neurosci. 2010; 30(36): 12005-12019, doi: 10.1523/JNEUROSCI.2208-10.2010, indexed in Pubmed: 20826664.

25. Patitucci TN, Ebert AD. SMN deficiency does not induce oxidative stress in SMA iPSC-derived astrocytes or motor neurons. Hum Mol Genet. 2016; 25(3): 514-523, doi: 10.1093/ hmg/ddv489, indexed in Pubmed: 26643950.

26. Satoh $M$, Fujimoto $S$, Haruna $Y$, et al. NAD(P)H oxidase and uncoupled nitric oxide synthase are major sources of glomerular superoxide in rats with experimental diabetic nephropathy. Am J Physiol Renal Physiol. 2005; 288(6): F1144-F1152, doi: 10.1152/ajprenal.00221.2004, indexed in Pubmed: 15687247.

27. Shababi M, Habibi J, Ma L, et al. Partial restoration of cardio-vascular defects in a rescued severe model of spinal muscular atrophy. J Mol Cell Cardiol. 2012; 52(5): 1074-1082, doi: 10.1016/j.yjmcc.2012.01.005, indexed in Pubmed: 22285962. 